\title{
Role of neuroimaging in multidisciplinary approach towards Non-Alzheimer's dementia
}

\author{
Satya Narayana Patro ${ }^{1} \cdot$ Rafael Glikstein $^{1} \cdot$ Prasad Hanagandi $^{1} \cdot$ Santanu Chakraborty $^{1}$
}

Received: 21 October 2014 / Revised: 7 July 2015 / Accepted: 8 July 2015 / Published online: 24 July 2015

(C) The Author(s) 2015. This article is published with open access at Springerlink.com

\begin{abstract}
Dementia is defined as chronic deterioration of intellectual function and cognitive skills significant enough to interfere with the ability to perform daily activities. Recent advances in the treatment of dementia have renewed interest in the use of various neuroimaging techniques that can assist in the diagnosis and differentiation of various subtypes. Neuroimaging and computational techniques have helped the radiological community to monitor disease progression of various neurodegenerative conditions presenting with dementia, such as Alzheimer disease, frontotemporal lobe dementia (FTLD), progressive supranuclear palsy (PSP) and multisystem atrophy-cerebellar variant (MSA-C), and their response to newer therapies. Prompt identification of treatable or reversible forms of dementia, such as tumours, subdural haemorrhage and intracranial dAVF, is crucial for the effective management of these conditions. It is also prudent to recognize the imaging spectrum of metabolic, infective and autoimmune diseases with rapidly progressing dementia, such as methanol toxicity, central pontine myelinolysis (CPM), delayed post hypoxic leukoencephalopathy (DPHL), HIV, Creutzfeldt-Jakob Disease (CJD), Sjogren's syndrome, multiple sclerosis (MS), radiation necrosis and Fragile X-Associated Tremor/Ataxia Syndrome (FXTAS), which are difficult to treat and often require palliative care. This pictorial review emphasizes various non-Alzheimer's dementia entities and discusses their imaging highlights.
\end{abstract}

Rafael Glikstein

rglikstein@toh.on.ca

1 Division of Neuroradiology, The Ottawa Hospital, University of Ottawa, 1053 Carling Avenue, K1Y 4E9 Ottawa, ON, Canada
Teaching Points

- Non Alzheimer's dementia constitutes a broad spectrum of conditions.

- Neuroimaging plays an important role in differentiating treatable from irreversible dementia.

- Neuroimaging is often non-specific in early stages of neurodegenerative conditions with dementia.

- Neuroimaging plays an important role in the multimodal approach towards management of dementia.

Keywords Dementia $\cdot$ Alzheimer · Atrophy $\cdot$ Myelinolysis Ataxia

\section{Introduction}

Dementia is a disorder characterized by global impairment in cognition, social and occupational functioning severe enough to interfere with daily functioning and quality of life. Various studies have confirmed an exponential increase in dementia with age and a higher prevalence in older females [1]. Overall, dementia has a significant burdening impact onsocioeconomic status and the health care system.

Alzheimer disease (AD) is the most common cause of primary dementia, constituting $60 \%$ of all cases. Vascular dementia is the second most common form of dementia, which often coexists with AD. There are various other nonAlzheimer conditions, which may present with cognitive impairments and can be divided into the broad categories of neurodegenerative, inflammatory/infective, metabolic/ genetic and miscellaneous. This pictorial review emphasizes the role of neuroimaging in evaluating various causes of nonAlzheimer's dementia. We also highlight conditions with reversible dementia, where neuroimaging plays a significant role in management. 


\section{Vascular dementia (VaD)}

Binswanger and Alzheimer first described VaD and recognized the role of multiple infarctions and chronic ischemia in its etiopathogenesis. VaD is the most common cause of nonAlzheimer dementia in the aging population and often contributes to cognitive impairment in $\mathrm{AD}$ and other forms of socalled "mixed dementias" [2]. Unlike AD, there are no pathological criteria for the diagnosis of $\mathrm{VaD}$. The clinical criteria are poorly understood and validated. It's a heterogeneous syndrome rather than a distinct disorder, in which the underlying cause is cerebrovascular disease in some form and its ultimate manifestation is dementia [3]. Three common forms of ischemic lesions can result in VaD: 1) Large artery infarcts involving the cortex and subcortical regions due to thromboembolic occlusion of major intracranial arteries; 2) Small artery infarctions or lacunes attributed to arteriolosclerosis involving the penetrating arteries and affecting the thalamus, basal ganglia, internal capsule, brain stem and cerebellum; 3) Periventricular white matter disease resulting from chronic subcortical ischemia of small arteries affecting the neurons, oligodedrocytes and astrocytes. Multiple studies have shown that in nearly 6 to $32 \%$ of cases with lobar and lacunar ischemic stroke, one can show post stroke cognitive impairment and dementia [4]. The presence of microbleeds, a manifestation of small vessel disease, is also associated with cognitive decline. The number of infarcts and anatomic distribution of ischemic stroke, rather than the volume, determines the development of $\mathrm{VaD}$. The more vulnerable areas are the hippocampus, angular gyrus, cingulate gyrus, frontal lobe or deep gray and white matter (thalamus, fornix, basal forebrain, caudate, globus pallidus, and the genu or anterior limb of the internal capsule) [5]. Advanced age, increased severity of the stroke, recurrent strokes, white matter disease, cortical atrophy (particularly in the temporal lobe), hypertension, obesity, elevated homocysteine, hyperlipidemia, and diabetes mellitus are the common associated risk factors [6]. The clinical presentation varies depending on cortical or subcortical ischemic lesions. Cortical lesions tend to be sudden onset with more focal neurodeficits, whereas subcortical lacunar infarcts and white matter disease have more gradual or stepwise decline of cognitive functions.

The diagnostic approach to patients with suspected $\mathrm{VaD}$ is similar to that of any other form of cognitive decline. Neuroimaging should be performed where there is history of stroke, vascular risk factors and clinical features atypical of AD. Magnetic resonance imaging (MRI) is considerably superior in sensitivity and specificity in detecting ischemic lesions compared to computed tomography (CT); however, radiological criteria are not adequate enough in differentiating between post-stroke patients with and without dementia [7]. Microangiopathic disease is the condition most commonly associated with vascular dementia and has non-specific imaging features. Confluent hypodensities in the periventricular and subcortical white matter (Fig. 1) with presence of cortical and lacunar infarcts are seen on CT. Likewise, MRI demonstrates confluent signal changes in the periventricular and subcortical white matter, deep grey nuclei and brain stem (Fig. 1). Increasing white matter lesion burden on serial MRI scans has been associated with accelerated cognitive decline in several studies [8]. Acute infarcts typically demonstrate T2W/FLAIR hyperintensity with diffusion restriction, whereas chronic infarcts exhibit volume loss with facilitated diffusion. GRE/SWI (Gradient Echo/Susceptibility Weighted Imaging) images are very sensitive in detecting microhaemorrhages noted in microangiopathic disease and other vascular conditions such as CADASIL (Cerebral Autosomal Dominant Arteriopathy with Subcortical Infarcts and Leukoencephalopathy), central nervous system (CNS) vasculitis and amyloid angiopathy. In summary, there are no uniform diagnostic criteria; however, a combination of clinical and neuropsychological assessment with neuroimaging inputs may help in the diagnosis and management of patients with $\mathrm{VaD}$.

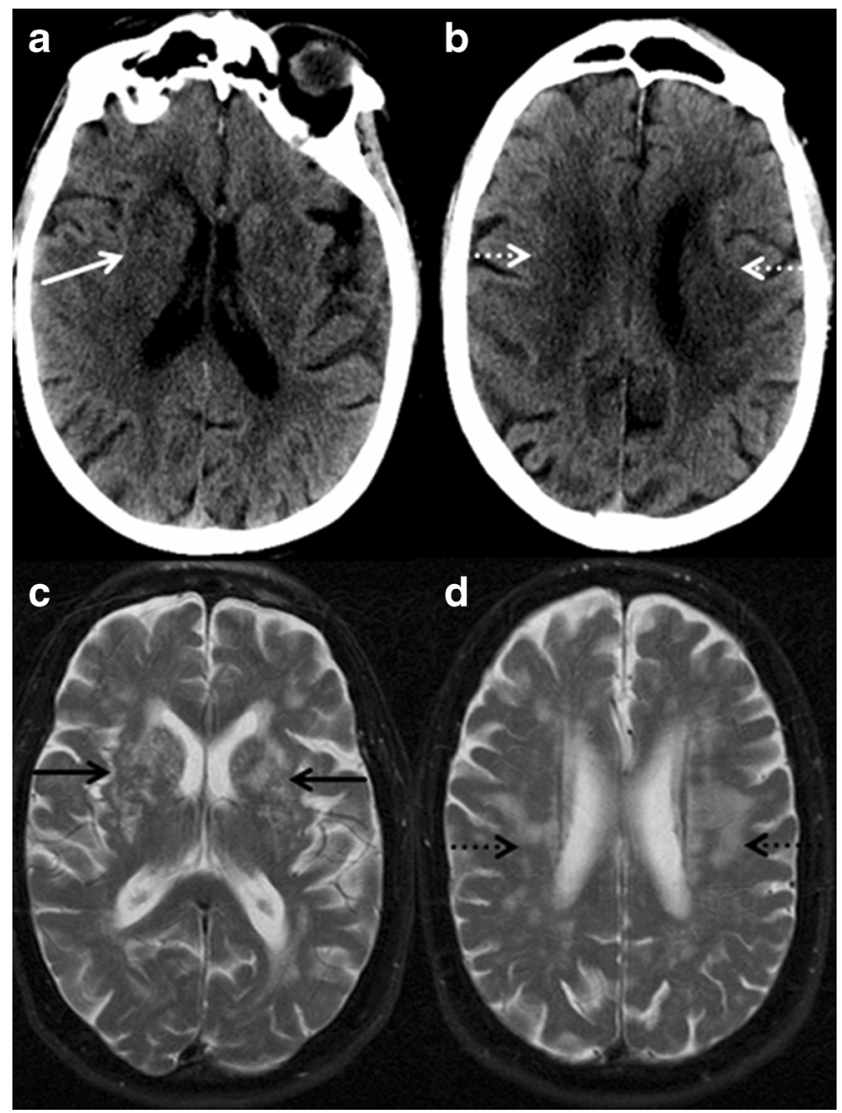

Fig. 1 Vascular dementia. An 86-year-old man with multiple transient ischemic attacks (TIAs), ongoing changes in level of consciousness and memory. a \& b noncontrast CT scan of the head shows multiple patchy confluent hypodensities in bilateral basal ganglia (white arrow), periventricular and subcortical white matter (dashed white arrow). c \& d T2W MRI images of the brain revealing diffuse patchy and confluent hyperintensities in bilateral basal ganglia (black arrows) and white matter (dashed black arrow) 


\section{Related conditions}

\section{CADASIL}

CADASIL is an inherited vasculopathy with subcortical dementia of Binswanger type. It affects the younger population, beginning in the fourth to fifth decade, and commonly presents with recurrent transient ischemic attacks, strokes, migraine and cognitive decline [9]. The etiopathogenesis is characteristic vasculopathy of small and medium size arteries. MRI is the most useful neuroimaging technique to demonstrate radiological picture of CADASIL. Symmetric and confluent T2W/FLAIR hyperintensities in periventricular and subcortical white matter are commonly demonstrated in CADASIL. Anterior temporal lobe white matter involvement is noted in nearly $90 \%$ of cases and uncommon in other microvascular diseases. Corpus callosum and insular T2W hyperintensities are also characteristic findings. DWI and GRE/SWI sequences are basically helpful in demonstrating occasional areas of associated acute infarcts and microhaemorrhages (Fig. 2) [10]. Brain atrophy is another important feature of CADASIL, likely resulting from subcortical ischemia and strongly correlated with cognitive decline.

\section{Chronic subdural hematoma (SDH)}

Chronic SDH is the most frequent cause of traumatic intracranial bleeding in the elderly population, especially in the background of cerebral atrophy. The mechanism of dementia can be explained by direct brain compression, reduction in whole brain cerebral blood flow $(\mathrm{CBF})$ and generalized astrocyte reactivity. Approximately $45 \%$ of patients with chronic $\mathrm{SDH}$ can have amnesia and cognitive deficits. This is considered to be one of the treatable forms of dementia and reversible upon surgical evacuation. On CT, chronic SDH often appears as a hypodense concavo-convex collection. On T1W and FLAIR MRI, it appears minimally hyperintense in comparison to CSF due to low paramagnetic methemoglobin and high protein content (Fig. 3). Membranes with loculations and varying stages of blood products should raise the suspicion of recurrent haemorrhage [11].

\section{Neurodegenerative}

\section{Dementia with Lewy bodies (DLB)}

Dementia with Lewy bodies (DLB) is considered as the second most common type of degenerative dementia after Alzheimer disease (AD). Cognitive dysfunction is the frequent initial presentation of DLB, with dementia eventually occurring in all cases. Unlike Alzheimer disease (AD), the patients with DLB often present with early impairments in attention, executive and visuospatial function, with memory affected later in the course of the disease [12]. Other clinical manifestations are visual hallucinations, dysautonomia, parkinsonism, sleep disorders, and neuroleptic sensitivity. Radiologic features are not specific, but may be supportive in the diagnosis of DLB. Generalized brain atrophy may be noticed
Fig. 2 CADASIL. A 51-year-old female with left hand weakness, slurred speech and dysarthria with progressive cognitive decline. a $\boldsymbol{\&} \mathbf{b}$ axial FLAIR and T2W images of brain demonstrate patchy confluent hyperintensities in periventricular (white dashed arrows) and subcortical white matter with typical involvement of temporal lobes (white arrow). c axial GRE image shows presence of microhaemorrhage in the subcortical white matter (black dotted arrow). d \& e axial DWI and ADC map of brain reveal focal area of restriction of diffusion (white arrow head) in the right corona radiata, suggesting acute infarct

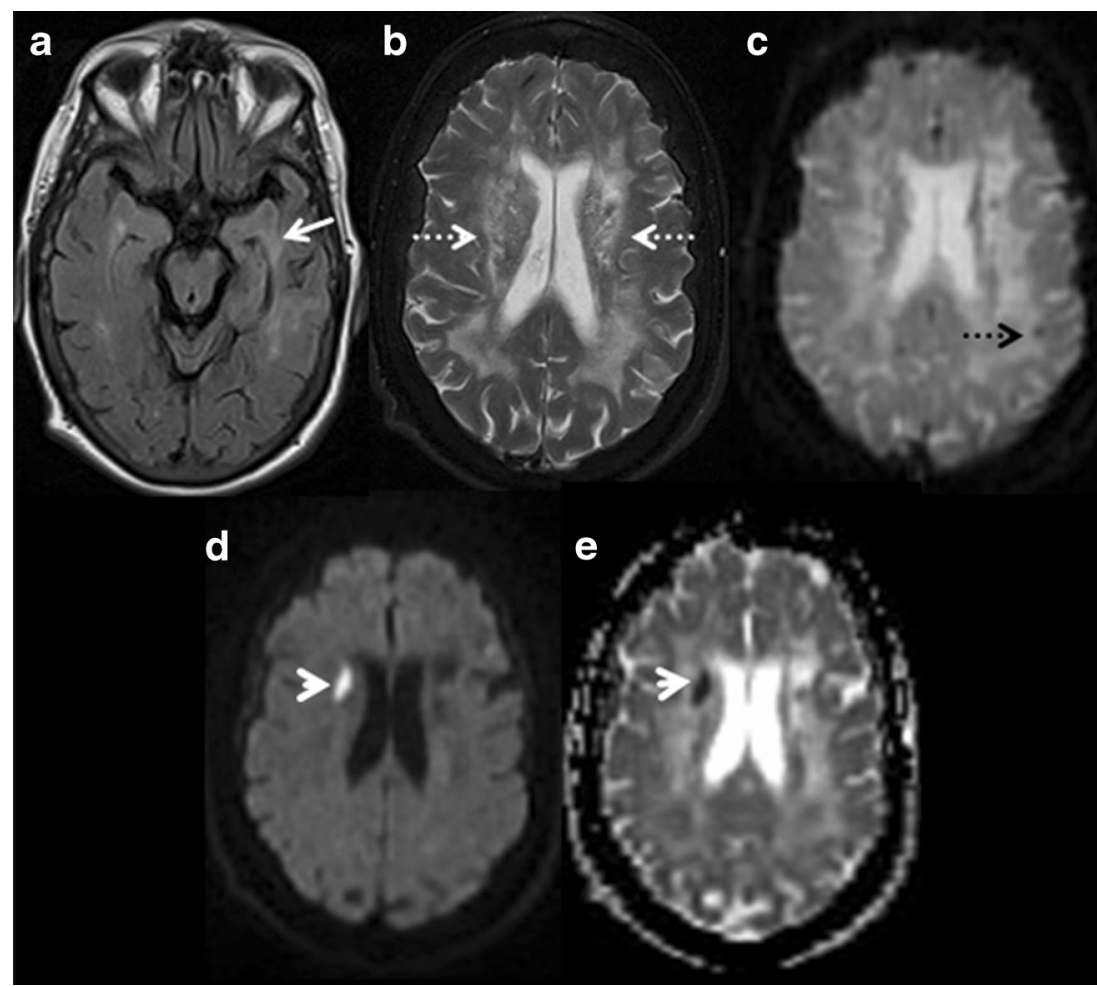




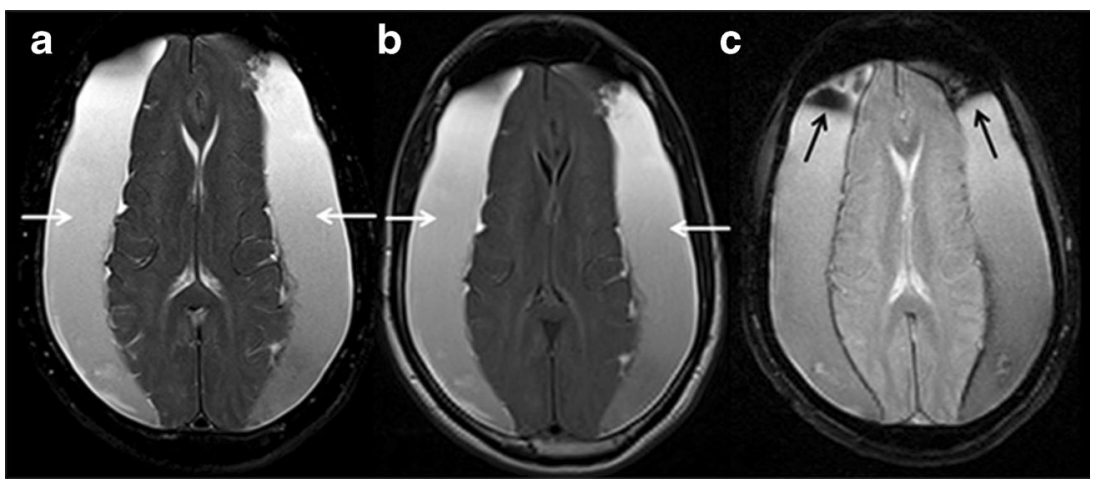

Fig. 3 Chronic subdural hematoma (SDH). A 73-year-old male with trivial head trauma later developed progressive loss of cognitive function. a \& b axial T2W and FLAIR sections of brain show hyperintense extra axial fluid collection in bilateral cerebral convexities (white arrows) with severe compression of adjacent cerebral hemispheres and effacement of the ventricles \& sulci. c axial GRE image of the brain demonstrate predominantly hyperintense fluid in bilateral cerebral convexities with hypointense margin on the medial aspect. There are also localized hypointensities (blooming susceptibility artefacts from the blood products) on the anterior aspects of the collections (black arrows) on MRI, similar to other neurodegenerative dementia. Few studies have shown atrophy of the putamen and dorsal mesopontine gray matter in DLB compared with AD on volumetric MRI scans [13]. As per one study, there can be reduced fractional anisotropy in the parieto-occipital white matter tracts on diffusion tensor imaging in DLB, but not in AD [14]. Single-photon emission computed tomography (SPECT) and positron emission tomography (PET) scans may show decreased perfusion and metabolism in the occipital areas [15]. The common differential diagnoses for DLB are Alzheimer disease (AD), Parkinson disease (PD), vascular dementia, other neurodegenerative dementias, and certain psychiatric disease. The diagnosis of DLB is primarily based on the clinical presentations, with radiologic features serving as helpful tool.

\section{Dementia in Parkinson disease (PD)}

Dementia is common and typically appears late in Parkinson disease (PD). Milder cognitive impairment represented by executive dysfunction and impaired visuospatial function is usually noted early in the disease [16]. Other associated clinical features are visual hallucinations, bradykinesia, rigidity and resting tremor. There are no specific imaging features for Parkinson disease with dementia; however, one of the recent neuroimaging studies has described the high accuracy of diagnosing PD by demonstrating absence of normal hyperintensity in the nigrosome-1 of the dorsolateral substantia nigra (Swallow Tail sign) on Susceptibility Weighted Imaging (SWI) MR sequence [17]. The most common differential diagnosis is Dementia with Lewy bodies (DLB). In PD, dementia generally occurs in the setting of wellestablished parkinsonism, whereas in DLB, dementia usually appears along with or before the development of parkinsonian signs. Quantitative morphometric MRI study can help in differentiating both entities with evidence of more pronounced cortical atrophy in DLB compared to PD. [18] Other differential diagnoses include progressive supranuclear palsy (PSP), corticobasal degeneration, Alzheimer disease and vascular dementia.

\section{Multiple system atrophy-cerebellar type (MSA-C)}

MSA-C was formerly called olivopontocerebellar degeneration, with onset at a mean age of 56 to 60 years. It is a sporadic disease with a combination of Parkinsonism symptoms, autonomic failure and cerebellar ataxia. Dementia is uncommon and of the frontal type, but is found in a minority of patients [19]. There is neuronal cell loss and gliosis in inferior olivary and pontine nuclei, cerebellar hemispheres and vermis. Common MR imaging features are selective atrophy of pons, middle cerebellar peduncles, cerebellar hemispheres and inferior olives (Fig. 4). An abnormal hyperintense signal that results from the degeneration of the transverse pontine fibres and pontine raphe gives the appearance of a "Hot Cross Bun" sign on T2W/FLAIR images (Fig. 4b) [20]. Although a nonspecific finding, it has also been observed in variant Creutzfeldt-Jakob disease, spinocerebellar atrophy types 2 and 3 and in parkinsonism secondary to vasculitis.

\section{Progressive supranuclear palsy (PSP)}

PSP is the most common form of atypical parkinsonian disorder, which can present with early executive dysfunction or subcortical dementia and clinically presents with supranuclear gaze palsy and motor symptoms. [21] Like FTLD, PSP is also related to abnormal tau protein deposition. Midbrain and superior cerebellar peduncle atrophy are classic neuroimaging features. Loss of normal superior midbrain tegmentum convexity with a relatively preserved pons on $\mathrm{T} 1 \mathrm{~W}$ mid-sagittal images gives the "hummingbird or penguin" beak appearance (Fig. 5a). Atrophy of superior cerebellar peduncles strongly 


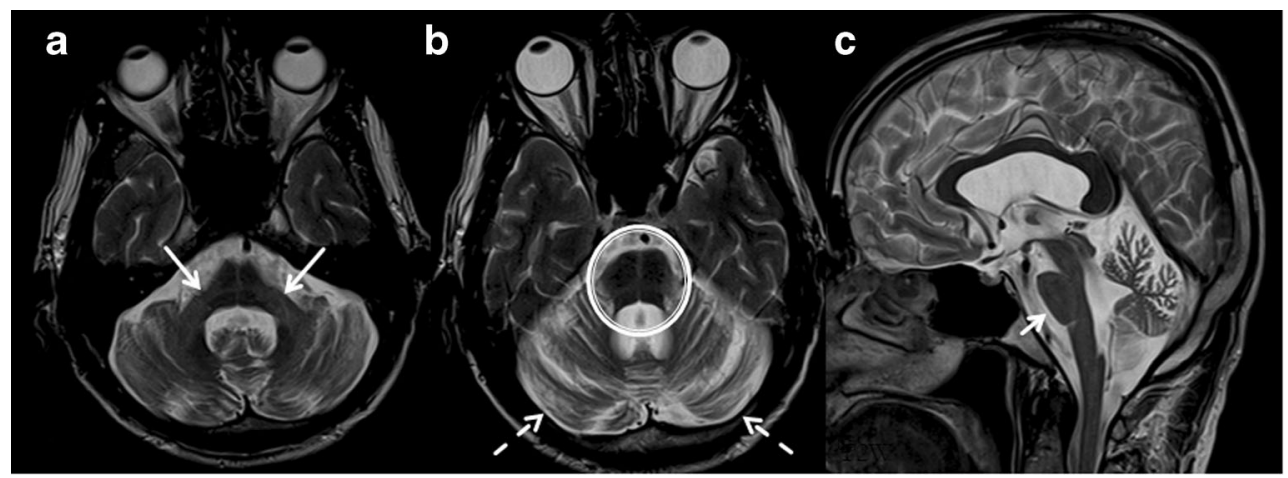

Fig. 4 Multisystem atrophy-cerebellar variant (MSA-C). A 79-year-old female with a two-year history of urinary incontinence, ataxia, rigidity, generalized atrophy and cognitive decline. a axial T2 W section of the posterior fossa shows atrophy of bilateral brachium pontis with hyperintense signal changes (white arrows). b axial T2W image at the level of

correlates with disease duration. Reduced anteroposterior midbrain diameter at the level of superior colliculus gives the "Mickey Mouse" appearance on axial images (Fig. 5c) [22]. Axial images may also show abnormal concavity of the lateral tegmental margin, giving the impression of a "morning glory" flower (Fig. 5b) [23]. A number of studies have used quantitative measurements to establish the specificity of atrophy pattern noted in PSP, and tried to differentiate from idiopathic parkinson's disease or MSA-P [24]. The anterior-posterior diameter and average area of the midbrain were found to be significantly lower in patients with PSP $\left(56 \mathrm{~mm}^{2}\right)$ compared to idiopathic Parkinson disease $\left(103 \mathrm{~mm}^{2}\right)$ or multiple system atrophy-parkinsonism (MSAP) $\left(97 \mathrm{~mm}^{2}\right)$ [25]. Similarly, PSP has shown a significantly higher ratio of the mid-sagittal pons to midbrain area compared with matched controls, multiple system atrophy or Parkinson disease. Few studies have suggested that magnetic resonance-parkinsonism index (MRPI) can be used to distinguish PSP from Parkinson disease and atypical parkinsonian syndrome [26]. It is an index calculated from the formula pons demonstrates typical hot cross bun sign (white circle) and significant atrophy and $\mathrm{T} 2 \mathrm{~W}$ hyperintensity in bilateral cerebellar hemispheres (white dashed arrows). $\mathbf{c}$ midline sagittal $\mathrm{T} 2 \mathrm{~W}$ image of brain is showing atrophy of pons mostly on the anterior aspect (short white arrow)

$(\mathrm{P} / \mathrm{M}) \times(\mathrm{MCP} / \mathrm{SCP})$, which requires measurement of the area of the pons $(\mathrm{P})$ and midbrain $(\mathrm{M})$ and width of the middle cerebellar peduncle (MCP) on sagittal T1-weighted MRI and the width of the superior cerebellar peduncle (SCP) on coronal MRI.

\section{Frontotemporal lobar dementia (FTLD)}

FTLD is a group of neurodegenerative diseases with a relatively rarer form of rapidly progressive dementia, characteristically involving the fronto-temporal lobes and insula. Clinically it comprises three subtypes: behavioral variant frontotemporal dementia (bvFTD), semantic dementia (SD) and nonfluent aphasia. The bvFTD is characterized by marked personality changes and behavioural problems, the most aggressive subtype with earliest average age of onset (fifth to sixth decade) and male preponderance [27]. SD or the temporal variant is also known as the least aggressive variant, and typically presents with either word finding difficulty or prosopagnosia (inability to recognize faces), depending on

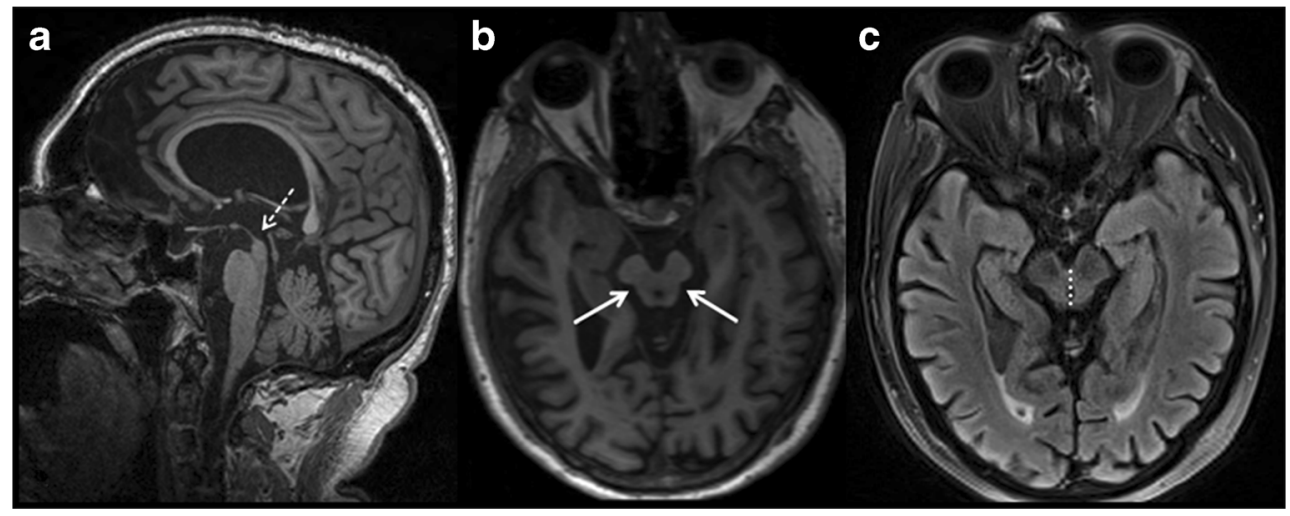

Fig. 5 Progressive Supranuclear Palsy (PSP). A 79-year-old male with gait ataxia and frequent falls with forgetfulness and loss of interest in routine activity. a sagittal T1W image of the brain shows loss of superior convexity of midbrain: "Humming bird or penguin sign" (white dashed arrow). $\mathbf{b}$ axial $\mathrm{T} 1 \mathrm{~W}$ section of the brain at the level of midbrain reveals abnormal concavity of lateral margin of midbrain: "morning glory sign" (white arrows). c axial FLAIR imaging of brain is demonstrating decreased anteroposterior diameter of midbrain (white dashed arrow) 
the predominant temporal lobe predilection [28]. Patients with non-fluent aphasia generally present with speech apraxia [29]. The characteristic neuropathological finding in FTLD is tau proteinopathy, as in AD. Neuroimaging is non-specific in the early phase of disease with overlap among subtypes, and is used primarily to exclude other forms of dementia. With clinical progression, FTLD subtypes frequently show specific patterns of focal cortical atrophy with the classic "knife edge" appearance involving frontal and/or temporal lobes. In the bvFTD subtype, bifrontal atrophy is the most common finding, which usually progresses to involve the temporal lobes at a later stage of the disease [30]. Behavioural changes are usually attributed to predominant involvement of the right frontal lobe (Fig. 6). The SD variant has predominant anterior temporal lobe atrophy and is more frequently marked on the left side, unlike $\mathrm{AD}$, in which the temporal lobe involvement tends to be symmetric with no antero-posterior gradient. Prominent atrophy of the left perisylvian region is characteristic of non-fluent aphasia [31]. Additionally, molecular imaging with either FDG-PET or rCBF (relative Cerebral Blood Flow) SPECT and quantitative analysis using 3D T1 MRI have shown good specificity in differentiating FTLD and $\mathrm{AD}$ based on identification of asymmetric and predominantly anterior versus posterior pattern of hypometabolism/perfusion and atrophy.

\section{Huntington's disease (HD)}

HD is an autosomal dominant, inherited progressive neurodegenerative disorder caused by a trinucleotide (CAG) repeat expansion in the huntingtin gene. It is characterized by abnormal choreiform movements, psychiatric symptoms and early onset dementia [32]. Cognitive deficits are inevitable in HD and common clinical manifestations are executive dysfunction, lack of insight and slowed processing. Caudate atrophy is the typical neuroimaging finding on axial MRI images (Fig. 7), and changes in linear caudate measurements correlate with severity of cognitive dysfunction [33]. Abnormal metabolic changes can also be noted on positron-emission tomography (PET) and functional MRI [34]. The differential diagnosis of HD is broad and ranges from benign familial chorea to hereditary and acquired neurodegenerative diseases. The diagnosis of HD is principally based upon a combination of clinical features, family history and presence of trinucleotide (CAG) repeat expansion in the HTT gene.

\section{Inflammatory/Infective}

\section{Creutzfeldt-Jakob disease (CJD)}

CJD is a fatal prion disease causing rapidly progressive dementia due to sub-acute spongiform encephalopathy. It commonly presents in the fifth to seventh decade of life with a triad of subacute dementia, myoclonus and characteristic triphasic EEG pattern. Elevated tau and/or 14-3-3 protein is seen on CSF analysis. MRI is the most suitable neuroimaging technique in the diagnosis of CJD, with T2W, FLAIR and DWI sequences being the most essential sequences of the MRI protocol. The key MRI features in CJD are T2W/ FLAIR hyperintensity in the caudate, putamen, anterior cingulate gyrus and thalamus. Widespread involvement of the cerebral cortex is also a characteristic feature [35]. DWI is very sensitive and shows diffusion restriction, especially in early stage of the disease, with no visible changes on $\mathrm{T} 2 \mathrm{~W} /$ FLAIR sequences (Fig. 8) [36]. Generalized atrophy is usually noted in late or terminal CJD. Variant CJD (vCJD) commonly presents with psychiatric symptoms and selective $\mathrm{T} 2 \mathrm{~W} /$ FLAIR hyperintensity with diffusion restriction in the medial and dorsal thalami (pulvinar), giving the appearance of "hockey-stick" or "pulvinar sign" [37]. A combination of clinical, neuroimaging and neuropathological findings frequently leads to the definitive diagnosis of CJD.

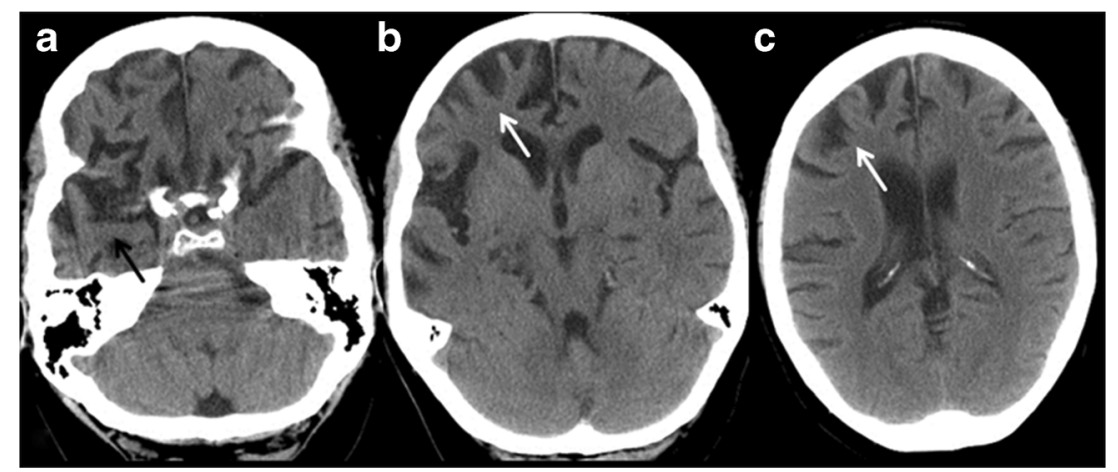

Fig. 6 Behavioural variant Frontotemporal dementia (bvFTD). A 53year-old male with progressive personality change and behavioural problems with cognitive decline. a, b \& c axial CT sections of brain show severe right frontal lobe atrophy (white arrow), as well as some atrophy of right anterior temporal lobe (black arrow) 


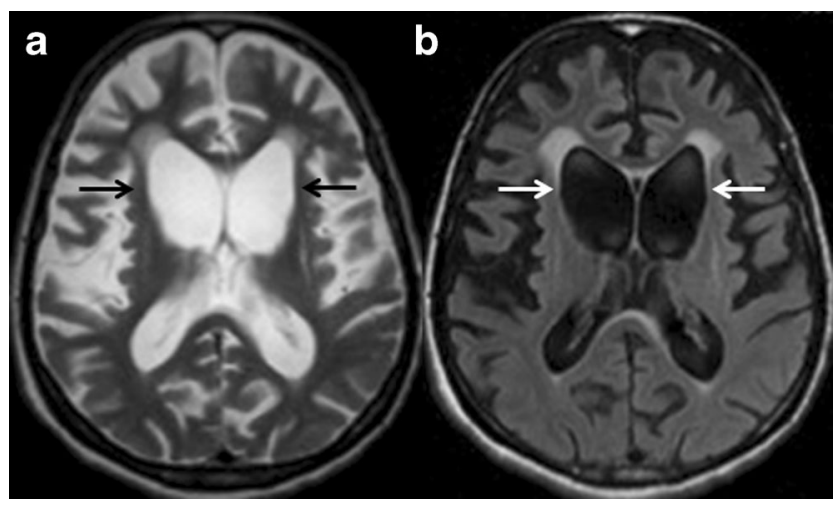

Fig. 7 Huntington's disease. A 62-year-old male presented with abnormal choreiform movement of the limbs, psychiatric manifestations and dementia. a axial T2W image at the level of lateral ventricles is showing significant atrophy of bilateral caudate nuclei (black arrows) and frontal lobes. b corresponding axial FLAIR image also demonstrates significant caudate atrophy (white arrows) and enlargement of frontal horns

\section{HIV-Associated dementia (HAD)}

Human Immunodeficiency Virus (HIV-1) has created a global crisis, with approximately 50 million people infected worldwide. HAD has also been previously referred to as AIDS dementia complex, HIV encephalitis or encephalopathy, and is seen in approximately $25-52 \%$ of patients with HIV infection, depending on disease state and anti-retroviral therapy. Patients develop insidious onset intellectual disturbance, psychomotor slowing and memory impairment. Generalized brain atrophy is the most common imaging finding. MRI may show patchy T2W/FLAIR hyperintensities in periventricular and subcortical white matter, which tends to be more diffuse and confluent with disease progression (Fig. 9) [38]. Lack of brain parenchymal or meningeal enhancement differentiates it from other opportunistic infections. Bilateral symmetrical hyperintensity in basal ganglia, thalami and brainstem has been described in the literature [39]. Advanced imaging techniques like magnetization transfer (MT) and proton MR spectroscopy (MRS) have shown low MT and decreased glutamate, NAA/choline ratios with increased myoinositol in the affected brain regions [39].

\section{Sjogren's syndrome (SS)}

Sjogren's syndrome is one of the most common autoimmune diseases, affecting the exocrine glands and manifesting as sicca syndrome. CNS involvement is noted in approximately $20 \%$ of patients, presenting with transverse myelitis and aseptic meningitis. It can manifest as steroidresponsive treatable dementia, which is caused by angiitis or nonvasculitic autoimmune inflammatory meningoencephalitis (NAIM). Cognitive and behavioural disorders like forgetfulness and bradyphrenia are some known presentations. MR imaging can often be normal or show nonspecific perivascular and subcortical small punctate white matter lesions (Fig. 10) [40, 41].
Fig. 8 Creutzfeldt-Jakob Disease (CJD). a FLAIR axial image of the brain shows hyperintensity in the right caudate head, putamen (white arrow) and occipital cortex (white dashed arrow). b \& c diffusion weighted image and ADC map reveal restriction of diffusion in the right caudate head, putamen and occipital cortex. $\mathbf{d} \&$ e diffusion weighted image and ADC map at higher level demonstrate restriction of diffusion in the right frontoparietal cortex

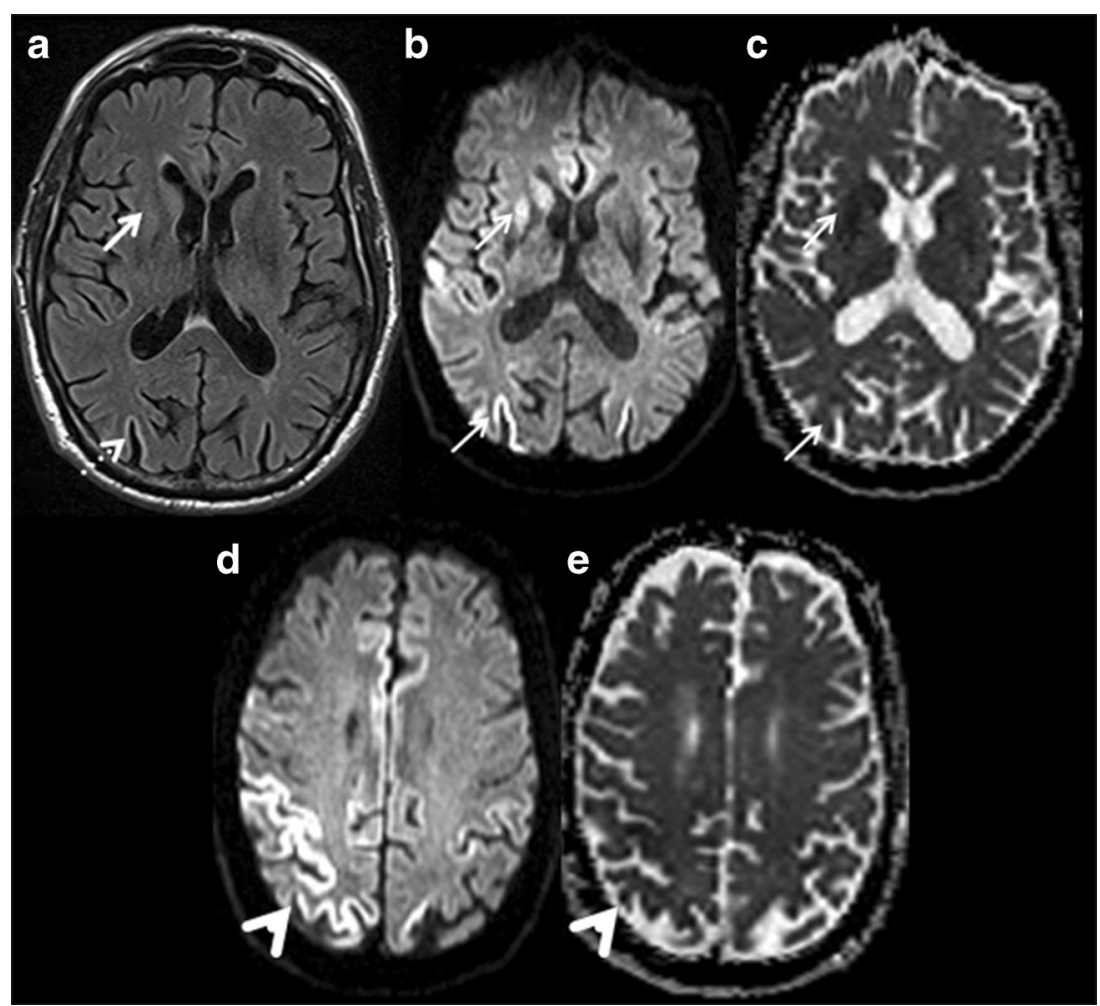




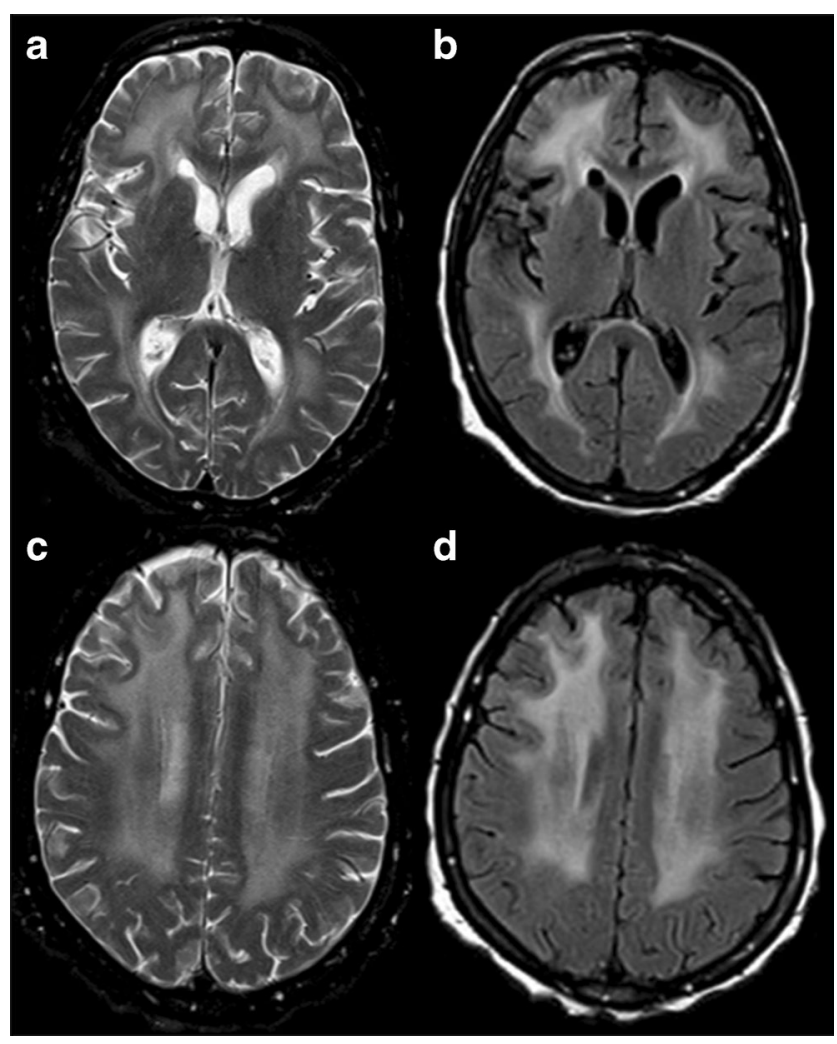

Fig. 9 HIV-Associated Dementia (HAD). A 56-year-old female with a several-month history of generalized weakness, confusion and memory loss. a \& b axial T2W and FLAIR images of the brain at the level of ventricles show diffuse symmetrical white matter hyperintensity and mild generalized atrophy. $\mathbf{c} \boldsymbol{\&} \mathbf{d}$ axial T2W and FLAIR images at the level of centrum semiovale reveal similar diffuse symmetrical hyperintensity in the white matter

\section{Multiple sclerosis (MS)}

MS is a demyelinating disease involving both white and gray matter. Focal neurological deficits caused by lesions in an eloquent long tract, like optic neuritis or a spinal cord

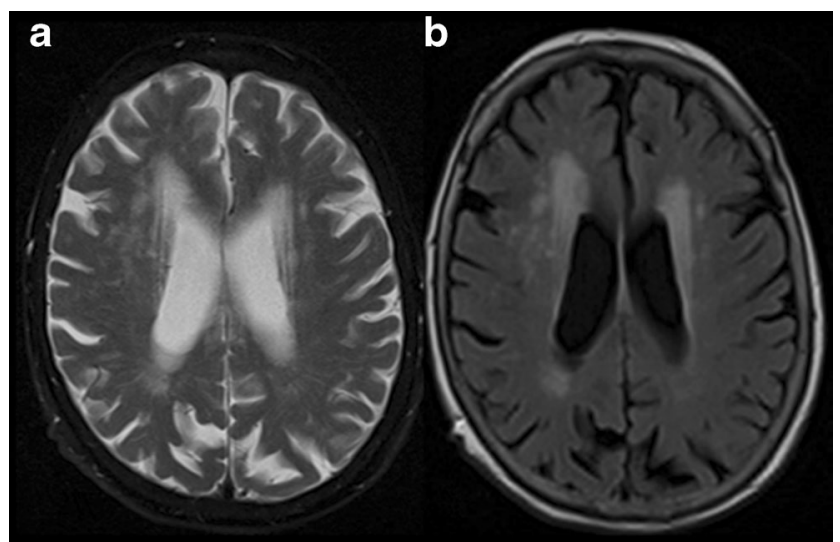

Fig. 10 Sjogren's syndrome. A 71-year-old female presented to hospital with unsteadiness, frequent falls and dementia with features of Sjogren's syndrome. a \& b axial T2W and FLAIR images of the brain show nonspecific patchy confluent hyperintensities in bilateral periventricular white matter syndrome, are common presentations. Nearly one in 20 patients can manifest with dementia and the common imaging finding is nonspecific generalized atrophy (Fig. 11) [42]. The degree of cognitive decline is proportional to the disease burden on MRI. Abnormal Magnetization Transfer Ratio (MTR) or Fractional Anisotropy (FA) have been reported in the cortex of MS patients with reduced NAA and increased $\mathrm{mI} / \mathrm{Cr}$ (mI- myo inositol, Cr- Creatine) ratios in deep grey matter on MRS, suggesting both axonal injury and gliosis [42].

\section{Metabolic/Genetic}

\section{Delayed post hypoxic leukoencephalopathy (DPHL)}

There are three basic mechanisms of developing hypoxicischemic brain injury- hypovolemia (cardiac failure), hypooxygenation (respiratory failure) and impaired oxygenation (CO poisoning) [43]. Initial clinical presentation includes a brief period of coma or altered consciousness. Recovery

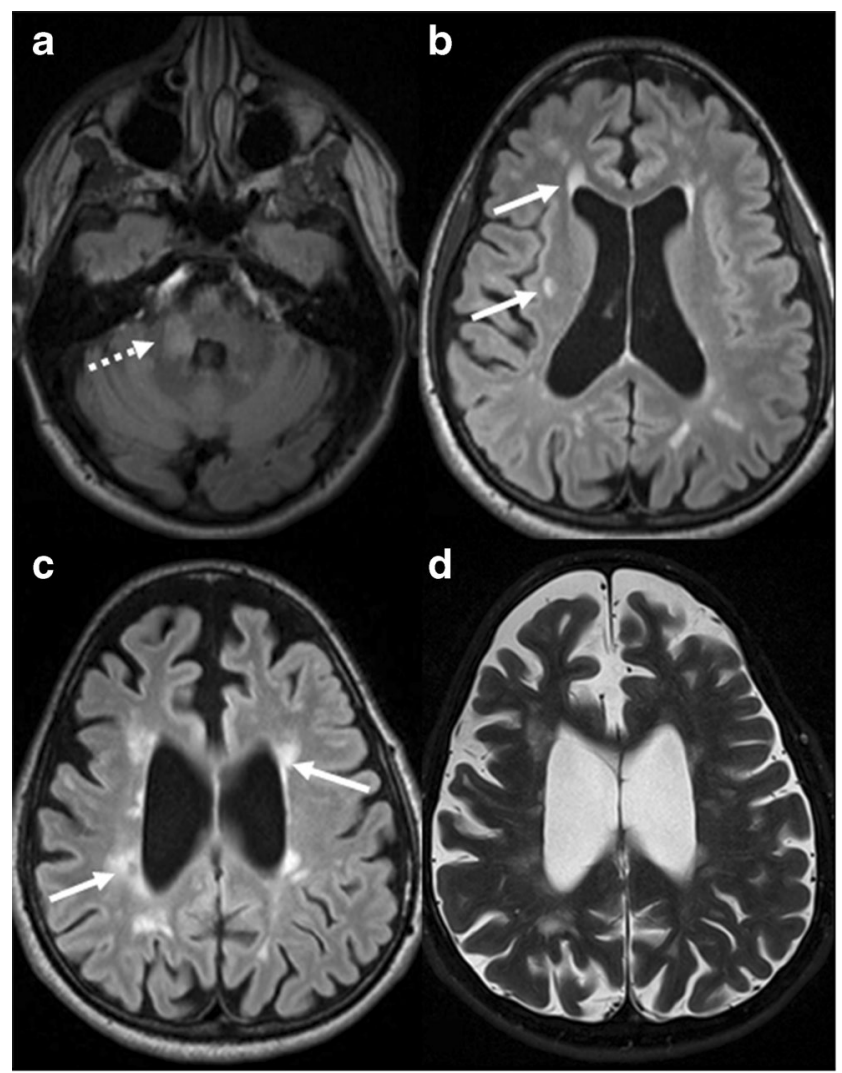

Fig. 11 Mutliple Sclerosis (MS). A 40-year-old female with history of relapsing remitting multiple sclerosis (RRMS) for the last 5 years, recently developed progressive memory decline. a axial FLAIR image of the posterior fossa shows a hyperintense demyelinating lesion in the right brachium pontis (white dashed arrow). b, c \& d axial FLAIR and T2W images at the level of lateral ventricles demonstrate multiple hyperintense lesions in periventricular white matter (white arrows) and generalized atrophy 
from the initial insult can manifest with short-term memory loss, confusion, ataxia and poor performance in executive function. Delayed post-hypoxic demyelination is considered to be the cause for cognitive deficits [44]. Diffuse cortical, periventricular white matter and deep gray matter T2W/FLAIR hyperintensity with DWI restriction are frequently noted on MRI (Fig. 12) [44]. The imaging appearance may overlap in few other conditions such as PRESS, spongiform leukoencephalopathy secondary to inhaled heroin and metachromatic leukodystrophy; however, history of hypoxia and cognitive decline are helpful in differentiating DPHL from other conditions.

\section{Acute methanol toxicity}

Methanol is a highly toxic, colourless liquid that resembles ethanol in taste and smell. Intoxication is common after suicidal or accidental ingestion and fraudulent adulteration of alcoholic beverages. It results in severe metabolic acidosis and optic necrosis or demyelination leading to vision loss. Severe toxicity may progress to Parkinson-like

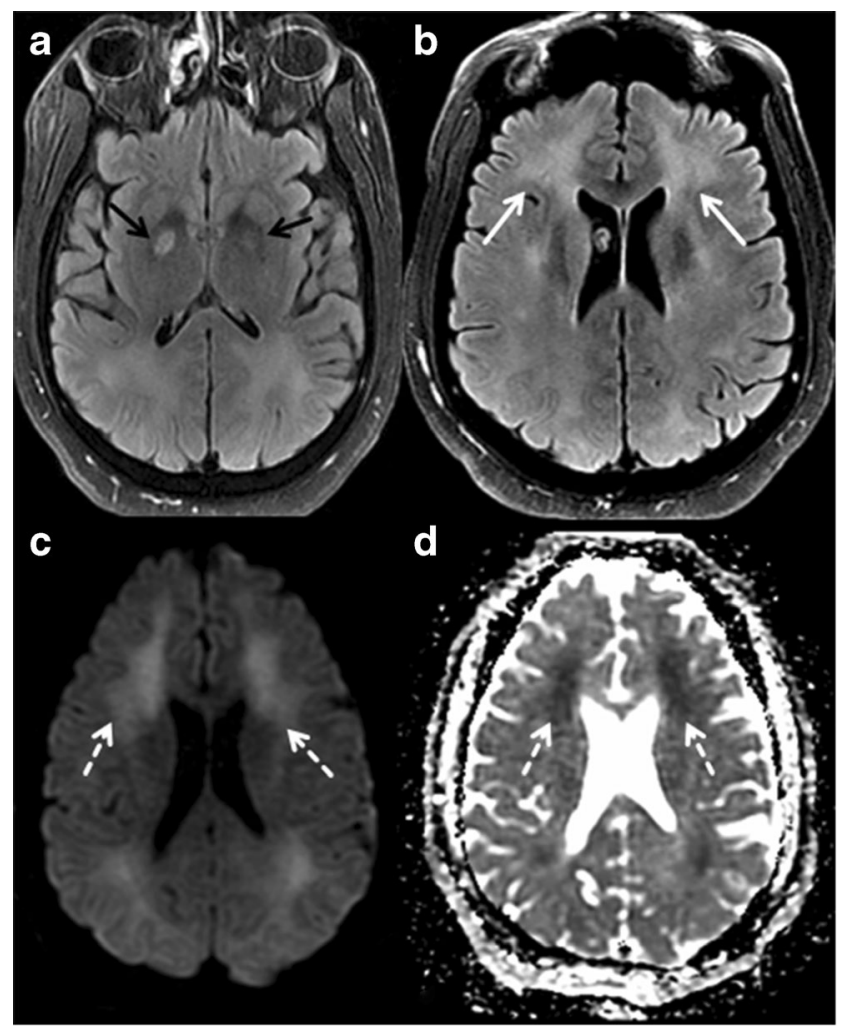

Fig. 12 Delayed Post Hypoxic Leukoencephalopathy (DPHL). A 42year-old male prisoner with history of substance abuse presented with altered level of consciousness, confusion, ataxia and memory loss. a axial FLAIR image is showing symmetrical necrosis of bilateral globus pallidus (black arrows). b axial FLAIR image at the level of ventricles demonstrates symmetrical periventricular and deep white matter hyperintensity (white arrow). $\mathbf{c} \& \mathbf{d}$ diffusion weighted image and ADC map reveal symmetrical restriction of diffusion in the deep white matter

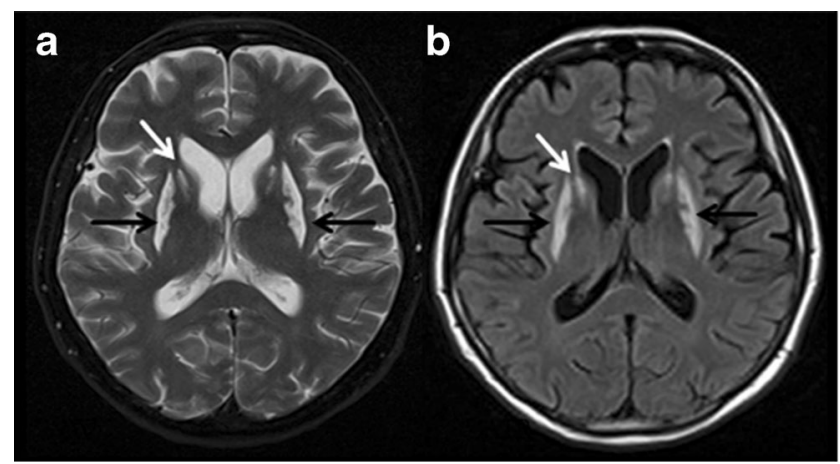

Fig. 13 Methanol toxicity. A 52-year-old female with a history of accidental methanol ingestion, presented with cognitive decline and rigidity in the extremities. $\mathbf{a} \boldsymbol{\&} \mathbf{b}$ axial T2W and FLAIR images of the brain show necrosis of bilateral putamen (black arrows) and head of caudate nuclei (white arrow)

extrapyramidal syndrome, cognitive deficits/dementia, permanent neurological deficits and death [45]. Both CT and MRI are helpful in demonstrating the changes in the brain. Bilateral putaminal necrosis with varying degrees of haemorrhage is the crucial imaging feature (Fig. 13). Cerebral and intraventricular haemorrhage, cerebellar and subcortical white matter necrosis and diffuse cerebral oedema are other common imaging manifestations [46]. Imaging differential diagnosis could include carbon monoxide poisoning, stroke, Wilson and Leigh disease.

\section{Central pontine myelinolysis}

Central pontine myelinolysis is an acute demyelinating process affecting the central pontine fibres and frequently associated with chronic alcoholism, nutritional deficiency and rapid correction of hyponatremia (osmotic demyelination). Spastic quadriparesis, pseudobulbar palsy, altered level of consciousness and coma are usual presentations. Dementia component and behavioural changes are likely from callosal involvement

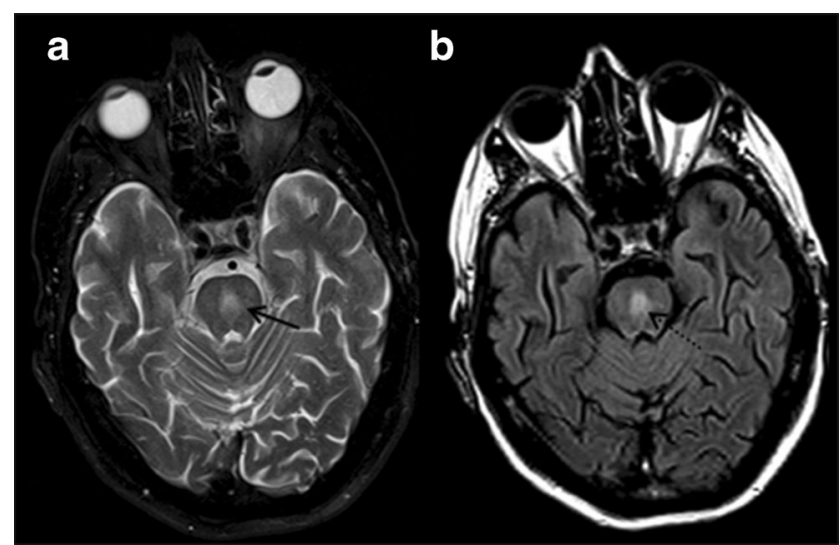

Fig. 14 Central Pontine Myelinolysis (CPM). A 61-year-old male with history of ETOH abuse with decrease in function, poor balance and dementia. a \& b axial T2W and FLAIR images at the level of pons show central pontine hyperintensity (black arrow) 


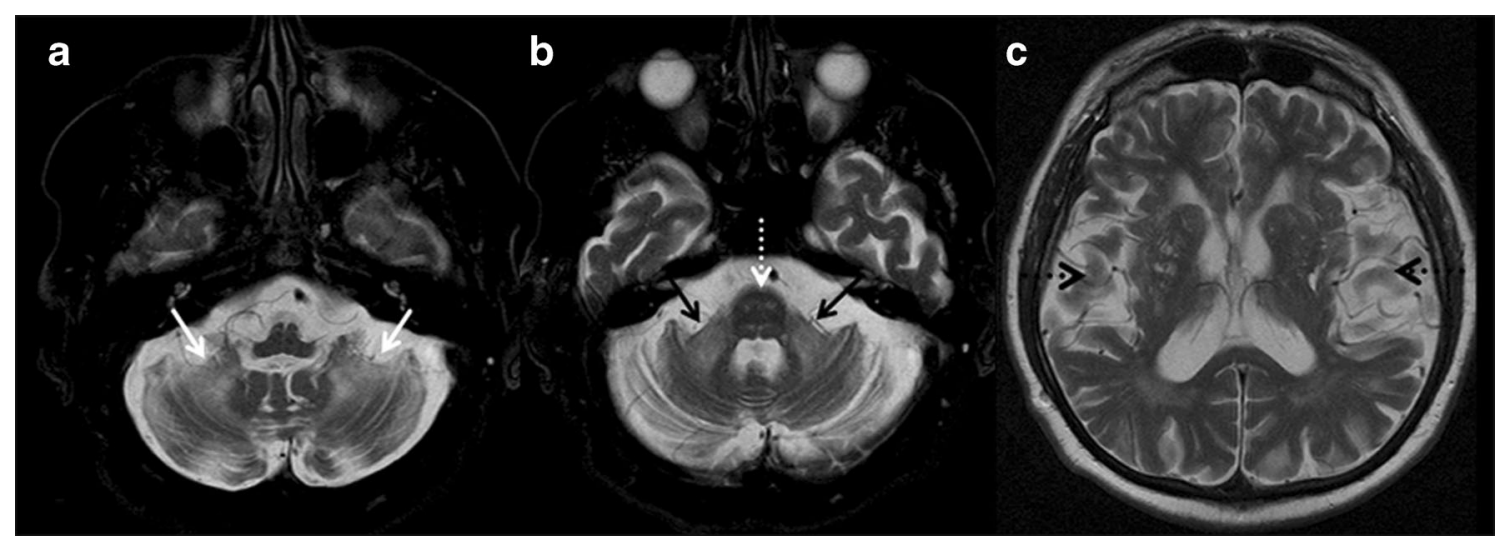

Fig. 15 Fragile X- Associated Tremor/Ataxia Syndrome (FXTAS). A 61-year-old male presented with a two-year history of tremor, rigidity in all four limbs, paresthesia with recent memory decline. a axial T2W image of the posterior fossa show atrophy and T2W hyperintensity in bilateral cerebellar hemisphere (white arrows). b axial T2W section of the brain at the level of pons demonstrate symmetrical hyperintensity in pons (dashed white arrow) and hyperintensity in bilateral middle cerebellar peduncles (black arrows). c axial T2W image of the brain at the level of lateral ventricles is revealing significant atrophy and T2W hyperintensities in bilateral cerebral hemispheres
[47]. Symmetric 'Trident shaped' T2W/FLAIR hyperintensity in the central pons with sparing of corticospinal tract are characteristic MR imaging features (Fig. 14). Diffusion restriction may be seen in the early phase with occasional peripheral post contrast enhancement [48]. Stroke, MS, encephalitis, glioma and toxic encephalopathy are common MR imaging differential diagnoses.

\section{Fragile X-associated tremor/Ataxia syndrome (FXTAS)}

FXTAS is a late onset $\mathrm{X}$ linked genetic cause of mental retardation and autism due to trinucleotide repeat mutation of the Fragile X Mental Retardation 1 (FMR1) gene, and is commonly seen in males between the ages of 50 and 80 years. The clinical presentation includes intentional tremor, ataxia, neuropathy, and personality changes in association with memory loss. Atrophy of the cerebral and cerebellar hemispheres, middle cerebellar peduncles, pons, medulla and corpus callosum are the important imaging features. Symmetric T2W/FLAIR hyperintense lesions in the cerebellar hemispheres close to the dentate nucleus, pons and bilateral middle cerebellar peduncles (MCP sign) are commonly noted (Fig. 15a \& b). In addition, symmetric patchy and/or confluent hyperintensities can be seen in periventricular and deep white matter of cerebral hemispheres with sparing of subcortical U fibers, cortical and deep gray matter and corpus callosum (Fig. 15c) [49].

\section{Miscellaneous}

\section{Intracranial dural AV fistula (dAVF)}

$\mathrm{dAVF}$ is an acquired pathological shunt between intracranial arteries and dural venous sinuses, meningeal or cortical veins.

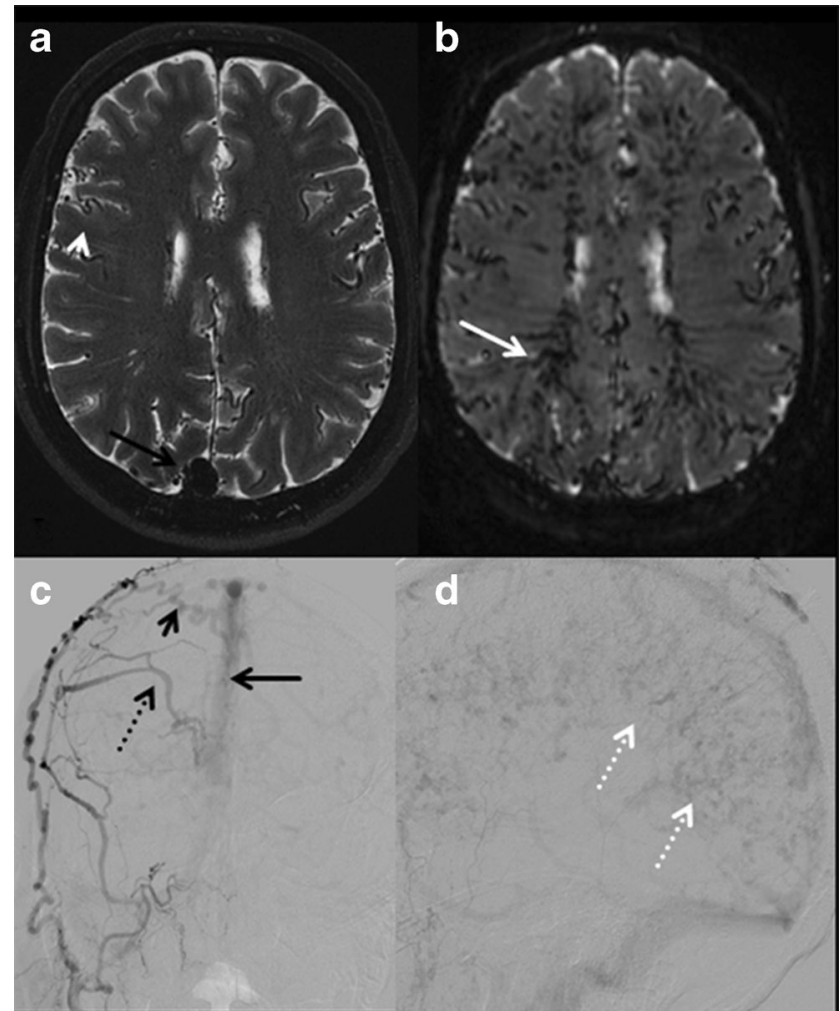

Fig. 16 Intracranial dural AV fistula. A 69-year-old male with a 1-month history of progressive cognitive decline. a $\&$ b axial T2W and GRE images of the brain show prominent superior sagittal sinus (black arrow) with multiple tortuous superficial cortical (arrow head) and medullary veins (white arrow). c right external carotid artery injection reveals dural AV fistula with abnormal communication between superior sagittal sinus (black arrow) and arterial feeders from right middle meningeal artery (dashed black arrow) and transosseous branches of right superficial temporal artery (short black arrow). d Late venous phase of the catheter angiogram shows prominent medullary venous drainage giving the appearance of pseudophlebitic appearance (dashed white arrows) 


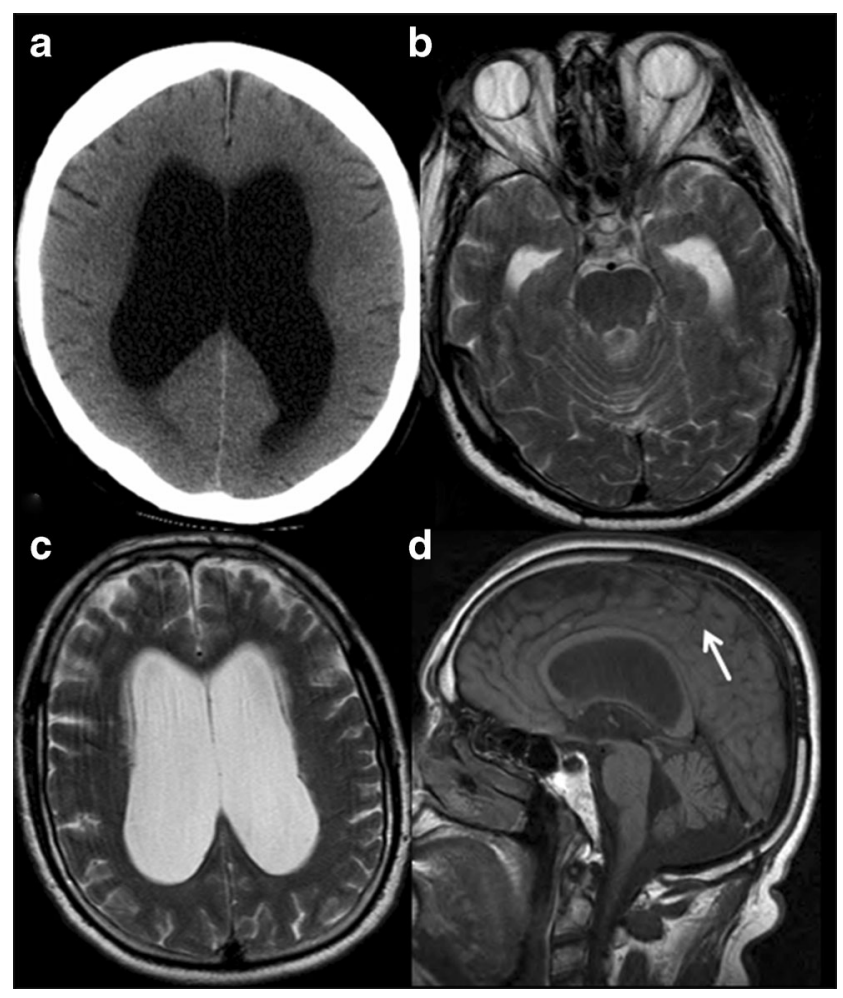

Fig. 17 Normal Pressure Hydrocephalus (NPH). A 75 -ear-old male presented with progressive gait ataxia and memory loss. a axial CT section of the brain shows dilated lateral ventricles with effaced sulci. b \& c axial T2W images of brain at the level of ventricles demonstrate dilatation of all the ventricles with prominent CSF flow void in the fourth ventricle (black arrow). d paramedian sagittal T1W section reveals ballooned corpus callosum with effacement of sulci towards vertex (white arrow)

It mostly presents in the fifth or sixth decades and the common locations are in transverse, sigmoid and cavernous sinuses [50]. Many dAVFs remain asymptomatic or have a benign course, but can present with cranial neuropathy, seizures, parkinsonism, cerebellar symptoms and intracranial haemorrhage in higher grade dAVFs. Cognitive decline and global dementia are uncommon clinical manifestations most likely resulting from venous hypertension and brain ischemia associated with impaired venous drainage [51]. Unenhanced CT is particularly limited to detecting intracranial haemorrhage and brain oedema. MRI is mainly suitable for demonstrating ectatic vessels, venous pouches, and signs of venous congestion such as white matter signal changes, haemorrhage, or venous infarcts (Fig. 16a and b). Dynamic CTA and time resolved MRA can be useful for demonstrating the dAVF and its relation to surrounding brain and skull, and thus may help in treatment planning. Digital subtraction angiogram is most accurate in demonstrating cranial dAVF, with superior visualization of arterial feeders, site of AV shunting and venous drainage (Fig. 16c \& d) [51]. Although a very rare cause of dementia, it can be reversible upon successful embolization of the fistula.

\section{Normal pressure hydrocephalus (NPH)}

Normal pressure hydrocephalus represents ventricular dilatation and clinical triad of cognitive decline, gait disturbance and urinary incontinence with normal CSF opening pressure. Management of this condition is a dilemma because of diagnostic uncertainty, and requires careful patient selection for CSF shunting. The aetiology is still debatable and explained by two existing theories: 1) Impaired CSF absorption due to prior meningitis or subarachnoid haemorrhage and 2) Decreased white matter tensile strength due to ischemic changes [52]. Imaging plays a minor role and MRI findings that may suggest NPH are 1) disproportionate ventricular dilatation in comparison to sulcal prominence; 2) periventricular halo suggesting transependymal CSF flow; and 3) prominent CSF flow void at the aqueduct of Sylvius, attributable to excessive rapidly pulsatile CSF flow [53]. Ballooning of the corpus callosum is another imaging feature (Fig. 17). MRI cine flow velocity mapping measures the aqueductal CSF flow velocity

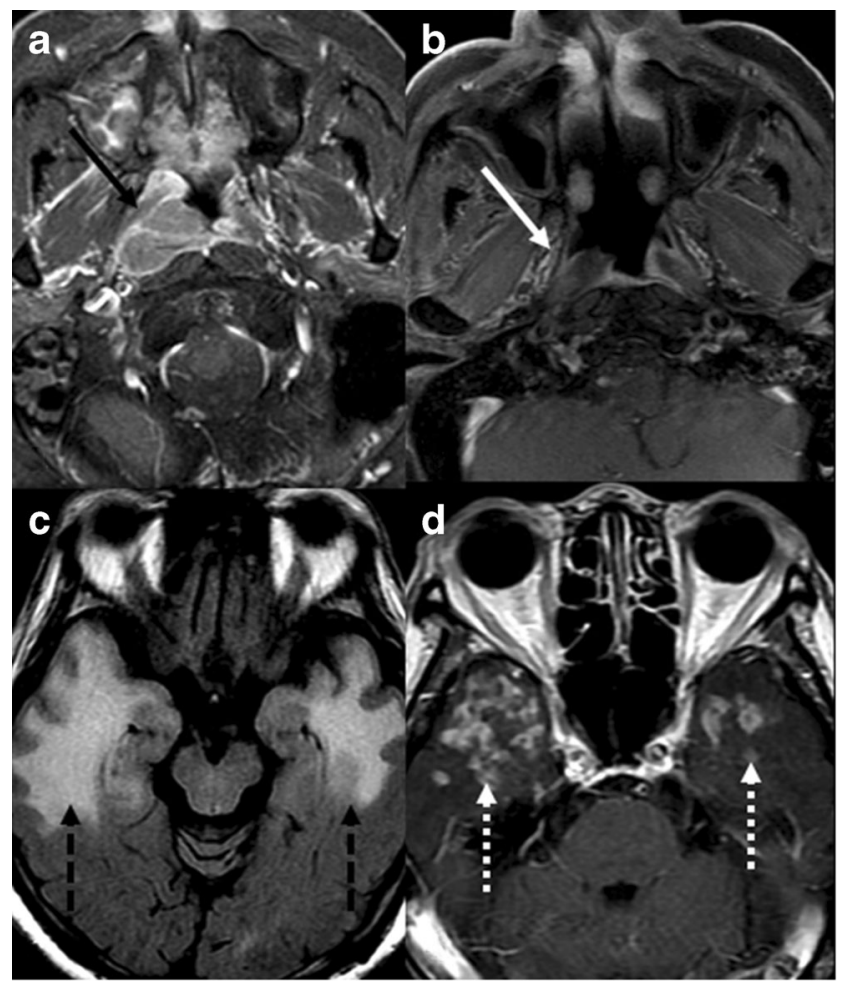

Fig. 18 Radiation necrosis with dementia. A 52-year-old male with history of nasopharyngeal carcinoma with radiotherapy developed progressive decline in cognition after 7 years of treatment. a axial post contrast T1W image at the level of skull base (2003) shows heterogeneously enhancing right side nasopharyngeal carcinoma (black arrow). b axial post contrast T1W image of the skull base (2007) reveals complete resolution of the mass lesion (white arrow). c \& d axial FLAIR and post contrast $\mathrm{T} 1 \mathrm{~W}$ images of the brain at the level of middle cranial fossa (2010) demonstrate extensive white matter hyperintensity (black arrows) and heterogeneous enhancement-"Swiss-cheese" pattern in bilateral temporal lobes (white arrows) 
and stroke volume is used at some centres to predict the response to shunt surgery.

\section{Radiation necrosis}

Patients irradiated for nasopharyngeal and oropharyngeal tumours can also present with cognitive and memory impairment due to bilateral temporal lobe involvement [54]. Radiation necrosis is a late complication occurring between 6 months to more than 10 years after radiotherapy. Diffuse T2/FLAIR hyperintense signal reflecting white matter oedema, cortical thinning and nodular, curvilinear or ring type post contrast enhancement are the common MRI features. The typical radiation necrosis enhancement has been described as a "Swiss-cheese" pattern (Fig. 18) [55]. MR perfusion and SPECT/PET studies may be useful in distinguishing viable tumour and radiation necrosis with low perfusion/ hypometabolism in the latter [55].

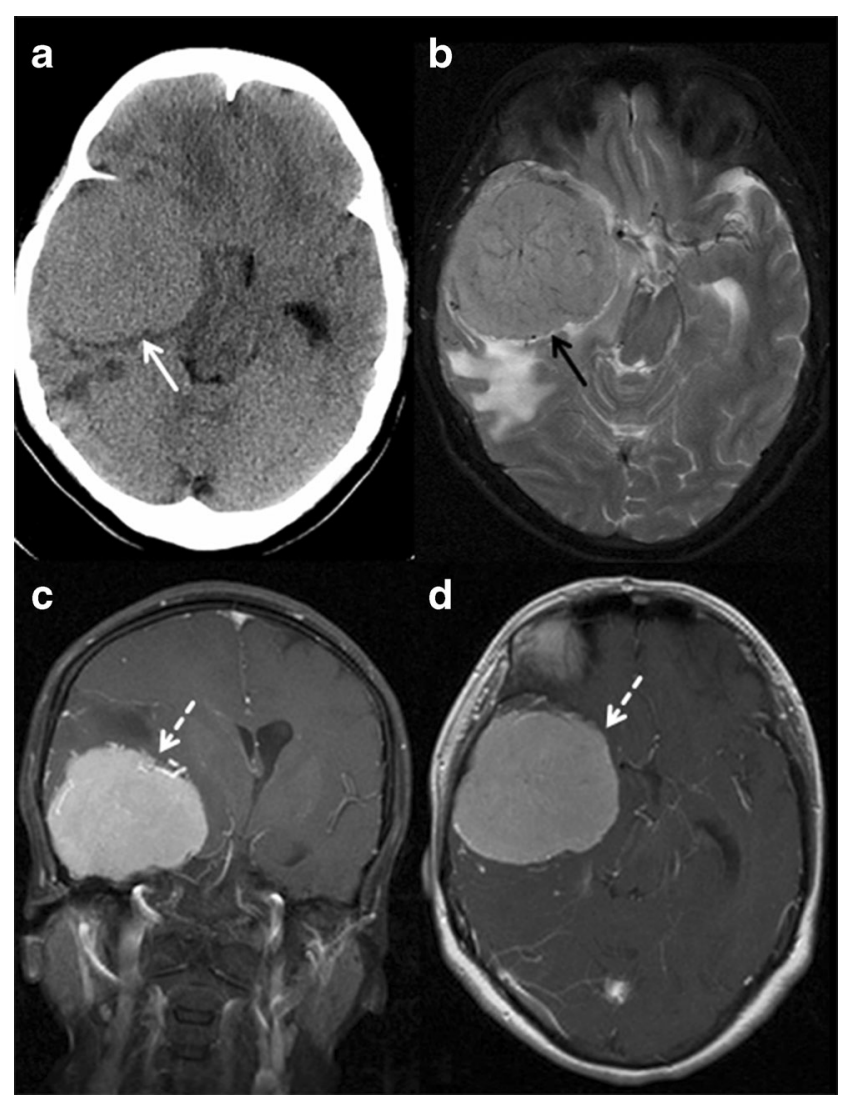

Fig. 19 Right middle cranial fossa meningioma. A 55-year-old female presented with LOC and memory disturbance. a axial noncontrast CT section of the brain shows large isodense mass lesion (white arrow) in the right middle cranial fossa with significant mass effect. b axial T2W image of the brain at the level of middle cranial fossa shows large extraaxial mass lesion (black arrow) in the right middle cranial fossa with perilesional oedema and compression of adjacent right temporal lobe. c $\boldsymbol{\&}$ d the lesion is showing homogeneous enhancement (white dashed arrow) on post contrast study

\section{Brain tumours}

Both extra and intra axial tumours, when situated near eloquent areas, may present with slowly progressing neuropsychological, behavioural disturbance and dementia. It is potentially a reversible cause of dementia if amenable to surgical resection. Common locations are being middle cranial fossa, basal frontal regions, thalamus, corpus callosum, cingulate gyrus and limbic system (Fig. 19). Neuroimaging aids in excluding tumoral pathologies in unexplained behavioural and cognitive disturbances [56].

\section{Conclusion}

Non-Alzheimer's dementia comprises several pathologies varying from rapid onset to gradually progressive disease spectrum. Often there is considerable clinical overlap, and neuroimaging features tend to be non-specific during the initial stage of disease. The rationale of conventional neuroimaging is not to offer definitive diagnoses, but to suggest possible differential diagnoses and track evolving changes on follow-up studies. Newer molecular imaging bio-markers combined with conventional neuroimaging techniques hold promise in assessing applications of novel diseasemodifying drugs for treatment and response evaluation. An important aspect of neuroimaging is also to recognize reversible or treatable causes of dementia that can potentially be treated or halt further disease progression.

Information on grants No grant or financial assistance were received from any source. The authors have no financial or intellectual conflict of interest.

Open Access This article is distributed under the terms of the Creative Commons Attribution 4.0 International License (http://creativecommons.org/licenses/by/4.0/), which permits unrestricted use, distribution, and reproduction in any medium, provided you give appropriate credit to the original author(s) and the source, provide a link to the Creative Commons license, and indicate if changes were made.

\section{References}

1. Prince M, Bryce R, Albanese E, Wimo A, Ribeiro W, Ferri CP (2013) The global prevalence of dementia: a systematic review and metaanalysis. Alzheimers Dement 9:63-75.e62

2. Roman GC (2002) Vascular dementia may be the most common form of dementia in the elderly. J Neurol Sci 203-204:7-10

3. Hachinski V, Iadecola C, Petersen RC et al (2006) National institute of neurological disorders and stroke-Canadian stroke network vascular cognitive impairment harmonization standards. Stroke 37: 2220-2241 
4. Kalaria RN (2012) Cerebrovascular disease and mechanisms of cognitive impairment: evidence from clinicopathological studies in humans. Stroke 43:2526-2534

5. Benisty S, Gouw AA, Porcher R et al (2009) Location of lacunar infarcts correlates with cognition in a sample of non-disabled subjects with age-related white-matter changes: the LADIS study. J Neurol Neurosurg Psychiatry 80:478-483

6. Srikanth VK, Quinn SJ, Donnan GA, Saling MM, Thrift AG (2006) Long-term cognitive transitions, rates of cognitive change, and predictors of incident dementia in a population-based first-ever stroke cohort. Stroke 37:2479-2483

7. Ballard CG, Burton EJ, Barber R et al (2004) NINDS AIREN neuroimaging criteria do not distinguish stroke patients with and without dementia. Neurology 63:983-988

8. Schmidt R, Petrovic K, Ropele S, Enzinger C, Fazekas F (2007) Progression of leukoaraiosis and cognition. Stroke 38:2619-2625

9. Ragno M, Tournier-Lasserve E, Fiori MG et al (1995) An Italian kindred with cerebral autosomal dominant arteriopathy with subcortical infarcts and leukoencephalopathy (CADASIL). Ann Neurol 38:231-236

10. Pantoni L, Pescini F, Nannucci S et al (2010) Comparison of clinical, familial, and MRI features of CADASIL and NOTCH3negative patients. Neurology 74:57-63

11. Fujisawa H, Nomura S, Kajiwara K, Kato S, Fujii M, Suzuki M (2006) Various magnetic resonance imaging patterns of chronic subdural hematomas: indicators of the pathogenesis? Neurol Med Chir (Tokyo) 46:333-338, discussion 338-339

12. Tiraboschi P, Salmon DP, Hansen LA, Hofstetter RC, Thal LJ, Corey-Bloom J (2006) What best differentiates Lewy body from Alzheimer's disease in early-stage dementia? Brain 129:729-735

13. Kantarci K, Ferman TJ, Boeve BF et al (2012) Focal atrophy on MRI and neuropathologic classification of dementia with Lewy bodies. Neurology 79:553-560

14. Watson R, Blamire AM, Colloby SJ et al (2012) Characterizing dementia with Lewy bodies by means of diffusion tensor imaging. Neurology 79:906-914

15. Mirzaei S, Rodrigues M, Koehn H, Knoll P, Bruecke T (2003) Metabolic impairment of brain metabolism in patients with Lewy body dementia. Eur J Neurol 10:573-575

16. Svenningsson P, Westman E, Ballard C, Aarsland D (2012) Cognitive impairment in patients with Parkinson's disease: diagnosis, biomarkers, and treatment. Lancet Neurol 11:697-707

17. Schwarz ST, Afzal M, Morgan PS, Bajaj N, Gowland PA, Auer DP (2014) The 'swallow tail' appearance of the healthy nigrosome - a new accurate test of Parkinson's disease: a case-control and retrospective cross-sectional MRI study at 3T. PLoS ONE 9:e93814

18. Beyer MK, Larsen JP, Aarsland D (2007) Gray matter atrophy in Parkinson disease with dementia and dementia with Lewy bodies. Neurology 69:747-754

19. Kawai Y, Suenaga M, Takeda A et al (2008) Cognitive impairments in multiple system atrophy: MSA-C vs MSA-P. Neurology 70: 1390-1396

20. Seppi K, Poewe W (2010) Brain magnetic resonance imaging techniques in the diagnosis of parkinsonian syndromes. Neuroimaging Clin N Am 20:29-55

21. McGinnis SM (2012) Neuroimaging in neurodegenerative dementias. Semin Neurol 32:347-360

22. Righini A, Antonini A, De Notaris R et al (2004) MR imaging of the superior profile of the midbrain: differential diagnosis between progressive supranuclear palsy and Parkinson disease. AJNR Am J Neuroradiol 25:927-932

23. Adachi M, Kawanami T, Ohshima H, Sugai Y, Hosoya T (2004) Morning glory sign: a particular MR finding in progressive supranuclear palsy. Magn Reson Med Sci 3:125-132

24. Hussl A, Mahlknecht P, Scherfler C et al (2010) Diagnostic accuracy of the magnetic resonance Parkinsonism index and the midbrain-to-pontine area ratio to differentiate progressive supranuclear palsy from Parkinson's disease and the Parkinson variant of multiple system atrophy. Mov Disord 25:2444-2449

25. Warmuth-Metz M, Naumann M, Csoti I, Solymosi L (2001) Measurement of the midbrain diameter on routine magnetic resonance imaging: a simple and accurate method of differentiating between Parkinson disease and progressive supranuclear palsy. Arch Neurol 58:1076-1079

26. Quattrone A, Nicoletti G, Messina D et al (2008) MR imaging index for differentiation of progressive supranuclear palsy from Parkinson disease and the Parkinson variant of multiple system atrophy. Radiology 246:214-221

27. Viskontas IV, Possin KL, Miller BL (2007) Symptoms of frontotemporal dementia provide insights into orbitofrontal cortex function and social behavior. Ann N Y Acad Sci 1121:528-545

28. Hodges JR, Patterson K, Ward R et al (1999) The differentiation of semantic dementia and frontal lobe dementia (temporal and frontal variants of frontotemporal dementia) from early Alzheimer's disease: a comparative neuropsychological study. Neuropsychology 13:31-40

29. Eslinger PJ, Dennis K, Moore P, Antani S, Hauck R, Grossman M (2005) Metacognitive deficits in frontotemporal dementia. J Neurol Neurosurg Psychiatry 76:1630-1635

30. Rankin KP, Gorno-Tempini ML, Allison SC et al (2006) Structural anatomy of empathy in neurodegenerative disease. Brain 129: 2945-2956

31. Gorno-Tempini ML, Dronkers NF, Rankin KP et al (2004) Cognition and anatomy in three variants of primary progressive aphasia. Ann Neurol 55:335-346

32. Walker FO (2007) Huntington's disease. Lancet 369:218-228

33. Bamford KA, Caine ED, Kido DK, Cox C, Shoulson I (1995) A prospective evaluation of cognitive decline in early Huntington's disease: functional and radiographic correlates. Neurology 45: 1867-1873

34. Young AB, Penney JB, Starosta-Rubinstein S et al (1986) PET scan investigations of Huntington's disease: cerebral metabolic correlates of neurological features and functional decline. Ann Neurol 20:296-303

35. Smart JM, Wood A (2001) Value of fluid-attenuated inversion recovery MR imaging in an unusual case of sporadic CreutzfeldtJakob disease. AJR Am J Roentgenol 177:948-949

36. Lee H, Hoffman C, Kingsley PB, Degnan A, Cohen O, Prohovnik I (2010) Enhanced detection of diffusion reductions in CreutzfeldtJakob disease at a higher B factor. AJNR Am J Neuroradiol 31:49 54

37. Vitali P, Maccagnano E, Caverzasi E et al (2011) Diffusionweighted MRI hyperintensity patterns differentiate CJD from other rapid dementias. Neurology 76:1711-1719

38. Thurnher MM, Thurnher SA, Fleischmann D et al (1997) Comparison of T2-weighted and fluid-attenuated inversion-recovery fast spin-echo MR sequences in intracerebral AIDS-associated disease. AJNR Am J Neuroradiol 18:1601-1609

39. Tucker KA, Robertson KR, Lin W et al (2004) Neuroimaging in human immunodeficiency virus infection. J Neuroimmunol 157: $153-162$

40. Alexander EL, Beall SS, Gordon B et al (1988) Magnetic resonance imaging of cerebral lesions in patients with the Sjogren syndrome. Ann Intern Med 108:815-823

41. Yoshikawa K, Hatate J, Toratani N et al (2012) Prevalence of Sjogren's syndrome with dementia in a memory clinic. J Neurol Sci 322:217-221

42. Anderson VM, Fox NC, Miller DH (2006) Magnetic resonance imaging measures of brain atrophy in multiple sclerosis. J Magn Reson Imaging 23:605-618

43. Shprecher D, Mehta L (2010) The syndrome of delayed posthypoxic leukoencephalopathy. NeuroRehabilitation 26:65-72 
44. Shprecher DR, Flanigan KM, Smith AG, Smith SM, Schenkenberg T, Steffens J (2008) Clinical and diagnostic features of delayed hypoxic leukoencephalopathy. J Neuropsychiatry Clin Neurosci 20:473-477

45. Jammalamadaka D, Raissi S (2010) Ethylene glycol, methanol and isopropyl alcohol intoxication. Am J Med Sci 339:276-281

46. Blanco M, Casado R, Vazquez F, Pumar JM (2006) CT and MR imaging findings in methanol intoxication. AJNR Am J Neuroradiol 27:452-454

47. Ghika-Schmid F, Ghika J, Assal G, Bogousslavsky J (1999) Callosal dementia: behavioral disorders related to central and extrapontine myelinolysis. Rev Neurol (Paris) 155:367-373

48. Ruzek KA, Campeau NG, Miller GM (2004) Early diagnosis of central pontine myelinolysis with diffusion-weighted imaging. AJNR Am J Neuroradiol 25:210-213

49. Brunberg JA, Jacquemont $\mathrm{S}$, Hagerman RJ et al (2002) Fragile $\mathrm{X}$ premutation carriers: characteristic MR imaging findings of adult male patients with progressive cerebellar and cognitive dysfunction. AJNR Am J Neuroradiol 23:1757-1766

50. Netravathi M, Pal PK, Bharath RD, Ravishankar S (2011) Intracranial dural arteriovenous fistula presenting as parkinsonism and cognitive dysfunction. J Clin Neurosci 18:138-140
51. Hurst RW, Bagley LJ, Galetta S et al (1998) Dementia resulting from dural arteriovenous fistulas: the pathologic findings of venous hypertensive encephalopathy. AJNR Am J Neuroradiol 19:12671273

52. Adams RD, Fisher CM, Hakim S, Ojemann RG, Sweet WH (1965) Symptomatic occult hydrocephalus with "normal" cerebrospinalfluid pressure. A treatable syndrome. N Engl J Med 273:117-126

53. Relkin N, Marmarou A, Klinge P, Bergsneider M, Black PM (2005) Diagnosing idiopathic normal-pressure hydrocephalus. Neurosurgery 57:S4-16; discussion ii-v

54. Lee PW, Hung BK, Woo EK, Tai PT, Choi DT (1989) Effects of radiation therapy on neuropsychological functioning in patients with nasopharyngeal carcinoma. J Neurol Neurosurg Psychiatry $52: 488-492$

55. Kumar AJ, Leeds NE, Fuller GN et al (2000) Malignant gliomas: MR imaging spectrum of radiation therapy- and chemotherapyinduced necrosis of the brain after treatment. Radiology 217:377384

56. Sahadevan S, Pang WS, Tan NJ, Choo GK, Tan CY (1997) Neuroimaging guidelines in cognitive impairment: lessons from 3 cases of meningiomas presenting as isolated dementia. Singap Med J 38:339-343 